\title{
Loss of menin in osteoblast lineage affects osteocyte- osteoclast crosstalk causing osteoporosis
}

\author{
Peng Liu ${ }^{1,2}$, Sooyeon Lee ${ }^{1,2}$, Jeanette Knoll ${ }^{2}$, Alexander Rauch ${ }^{2,9}$, Susanne Ostermay ${ }^{2}$, Julia Luther ${ }^{3}$, Nicole Malkusch ${ }^{1}$, Ulf H Lerner ${ }^{4}$, \\ Mario M Zaiss ${ }^{5}$, Mona Neven ${ }^{3}$, Rainer Wittig ${ }^{6}$, Martina Rauner ${ }^{7}$, Jean-Pierre David ${ }^{3,5}$, Philippe Bertolino ${ }^{8}$, Chang X Zhang ${ }^{8}$ and \\ Jan P Tuckermann ${ }^{*, 1,2}$
}

During osteoporosis bone formation by osteoblasts is reduced and/or bone resorption by osteoclasts is enhanced. Currently, only a few factors have been identified in the regulation of bone integrity by osteoblast-derived osteocytes. In this study, we show that specific disruption of menin, encoded by multiple endocrine neoplasia type 1 (Men1), in osteoblasts and osteocytes caused osteoporosis despite the preservation of osteoblast differentiation and the bone formation rate. Instead, an increase in osteoclast numbers and bone resorption was detected that persisted even when the deletion of Men1 was restricted to osteocytes. We demonstrate that isolated Men1-deficient osteocytes expressed numerous soluble mediators, such as C-X-C motif chemokine 10 (CXCL10), and that CXCL10-mediated osteoclastogenesis was reduced by CXCL10-neutralizing antibodies. Collectively, our data reveal a novel role for Men1 in osteocyte-osteoclast crosstalk by controlling osteoclastogenesis through the action of soluble factors. A role for Men1 in maintaining bone integrity and thereby preventing osteoporosis is proposed.

Cell Death and Differentiation (2017) 24, 672-682; doi:10.1038/cdd.2016.165; published online 20 January 2017

The maintenance of bone mass is severely affected in osteoporotic patients and other bone disorders and can have multiple etiologies. The complex communication of boneforming osteoblasts, bone-residing osteocytes, and boneresorbing osteoclasts is still not fully understood. Two hallmark studies $^{1,2}$ have demonstrated that osteocytes are the major source of the osteoclastogenesis-stimulating factor RANKL and thus key components in the control of bone mass. This property goes beyond their previously suggested function of sensing the mechanical loading of bone. ${ }^{3} \mathrm{PTH}^{4}$ and sclerostin ${ }^{5}$ have been reported to stimulate osteocytes to support osteoclastogenesis via a RANKL-dependent pathway. High-mobility group box 1 (HMGB1), which is chemotactic to osteoclasts, is enhanced in apoptotic MLO-Y4 cells; ${ }^{6}$ however, there is little information on other factors controlling osteoclast activity by osteoblast-derived osteocytes.

The nuclear protein menin encoded by the gene Men1 (multiple endocrine neoplasia type 1) has recently been suggested to control bone mass at higher age through its influence on the differentiation and function of osteoblasts. ${ }^{7}$ In vitro studies in cell lines and primary osteoblasts derived from 6-month-old mice showed that menin promotes early osteoblast differentiation in committed cells. Knockdown of Men1 with antisense oligonucleotides lead to enhanced BMP2 signaling ${ }^{8-10}$ and to facilitated inhibition of the late stage of osteoblast differentiation by potentiating TGF $\beta$-dependent Smad3 signaling. ${ }^{11,12}$ Accordingly, reduced bone mass and reduced bone formation due to decreased osteoblast proliferation and differentiation and enhanced apoptosis have been reported using aged osteocalcin-cre mice crossed to Men 1 floxed mice that leads to a conditional deletion of menin in the late stage of differentiated osteoblasts. ${ }^{7}$ On the other hand, aged osteoblast-specific menin transgenic mice were found to display increased bone mass. ${ }^{7}$ However, whether Men 1 plays a role in bone cells to control bone integrity in young or middle-aged mice is completely unknown.

Using multiple cre-lines, we comprehensively analyzed the effects of loss of function of menin throughout the osteoblast lineage, in osteoclasts, and in osteocytes on bone integrity. Furthermore, we identified genes under the control of Men1 responsible for the osteocyte-dependent regulation of osteoclast formation.

\section{Results}

Men1 disruption in the osteoblast lineage severely affects bone mass but not the bone formation rate. In order to address the role of Men1 in the entire osteoblast lineage including osteocytes in vivo, we generated mice lacking Men1 specifically in the osteoblast lineage by

${ }^{1}$ Institute of Comparative Molecular Endocrinology, University of Ulm, Ulm D-89081, Germany; ${ }^{2}$ Tissue-specific Hormone Action, Leibniz Institute on Aging-Fritz Lipmann Institute (FLI), Jena D-07745, Germany; ${ }^{3}$ Department of Osteology and Biomechanics, University Medical Center Hamburg-Eppendorf, Hamburg D-20246, Germany; ${ }^{4}$ Centre for Bone and Arthritis Research, Department of Internal Medicine and Clinical Nutrition at Institute for Medicine, Sahlgrenska Academy at University of Gothenburg, Gothenburg SE-41345, Sweden; ${ }^{5}$ Department of Internal Medicine 3-Rheumatology and Immunology, Friedrich-Alexander-University Erlangen-Nürnberg (FAU) and Universitätsklinikum Erlangen, Erlangen D-91054, Germany; ${ }^{6}$ Institute for Laser Technologies in Medicine and Metrology at Ulm University, Ulm D-89081, Germany; ${ }^{7}$ Division of Endocrinology and Bone Diseases, Department of Medicine III, TU Dresden, Dresden D-01307, Germany and ${ }^{8}$ Centre de Recherche en Cancérologie de Lyon, Inserm U1052, CNRS UMR5286, Université Lyon 1, Lyon F-69000, France

*Corresponding author: JP Tuckermann, Institute of Comparative Molecular Endocrinology, University of Ulm, Ulm D-89081, Germany. Tel: +49 731 50 $32600 ;$ Fax: +49 731 50 32609; E-mail: jan.tuckermann @uni-ulm.de

${ }^{9}$ Current address: Department of Biochemistry and Molecular Biology, University of Southern Denmark, DK-5230 Odense, Denmark.

Received 30.6.16; revised 08.12.16; accepted 20.12.16; Edited by E Wagner; published online 20.1.2017 
a

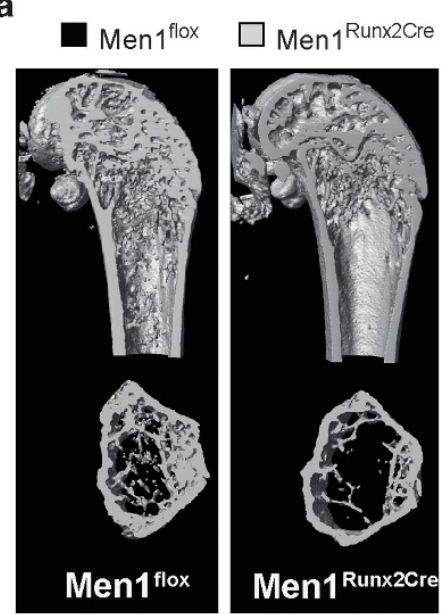

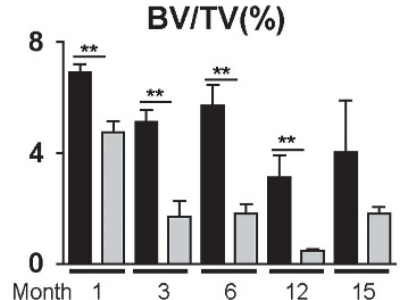

d

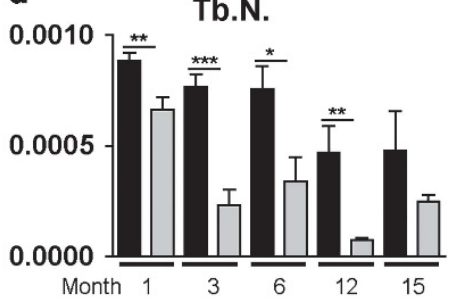

c

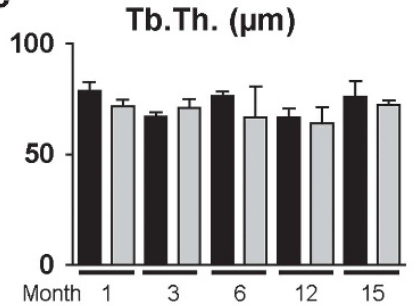

e

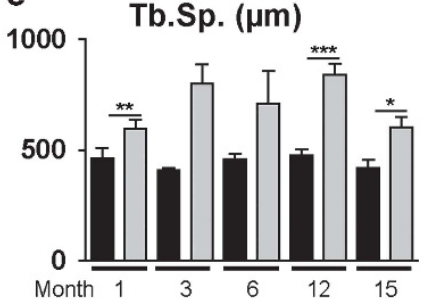

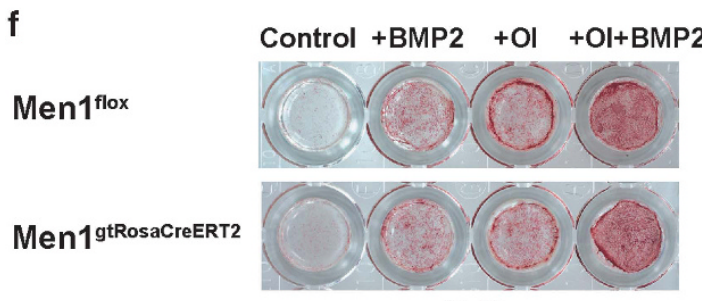

ALP

\section{g}

Men1 $1^{\text {flox }}$

Men1 $1^{\text {gtRosaCreERT2 }}$

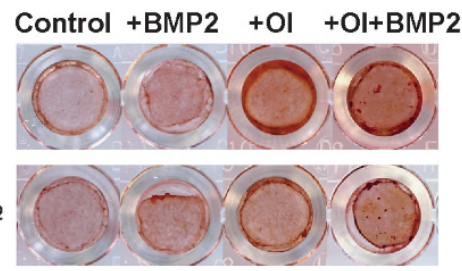

Alizarin red
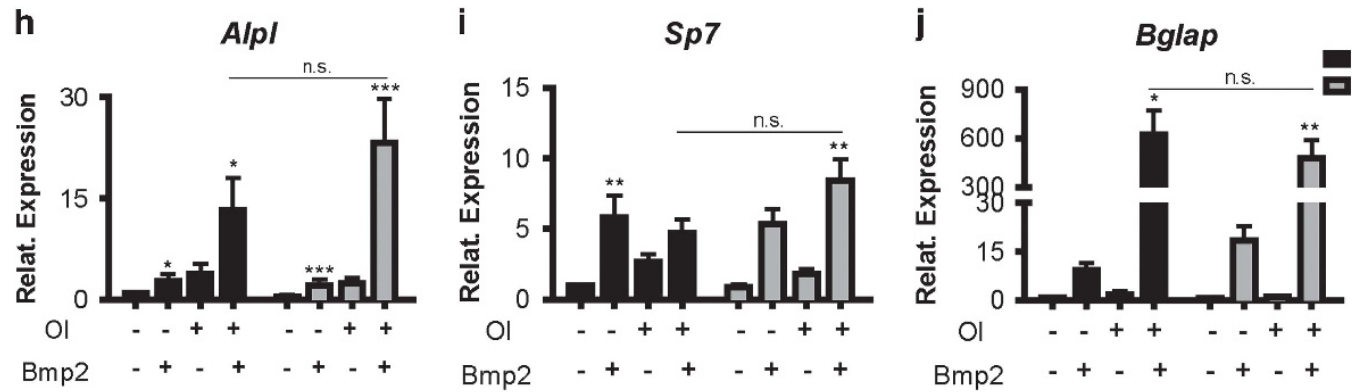

$\mathbf{K}$
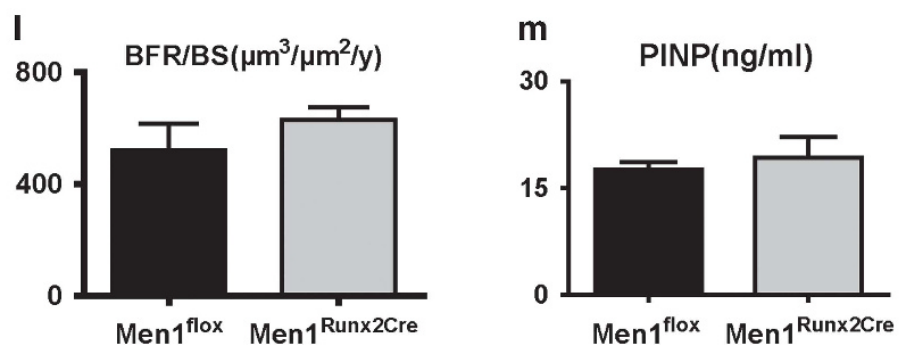

Figure 1 Disrupting Men1 in the osteoblast lineage causes bone loss without altering the bone formation rate. (a) Micro CT reconstruction of femurs from 12-week-old female Men $1^{\text {flox }}$ and Men $1^{\text {Runx2Cre }}$ mice. (b-e) Cancellous parameters such as bone volume per tissue volume (BV/TV) (b), trabecular thickness (Tb.Th.) (c), trabecular numbers (Tb.N.) (d), and trabecular separation (Tb.Sp.) (e) were measured from distal femurs of female Men $f^{\text {tlox }}$ and Men $1^{\text {Runx2Cre }}$ mice at indicated age by micro CT ( $n=4$ or 5 ). ${ }^{*} P<0.05$, ${ }^{* *} P<0.01,{ }^{* * *} P<0.001$. (f and $\mathbf{g}$ ) Primary calvarial osteoblasts were isolated from Men $f^{\text {flox }}$ and Men $1^{\text {gtRosaCreERT2 }}$ mice. After pretreatment with 4-OHT to allow recombination of the Men1flox allele, cells were cultured with or without osteogenic induction (OI) medium in the absence or presence of BMP2 for 7 and 12 days and stained for alkaline phosphatase (f) and alizarin red (g), respectively. (h-j) mRNA expression levels of $A / p /(\mathbf{h})$ and $S p 7$ (i) from 7-day-treated cells as in (f), and Bglap (j) from 12-day-treated cells as in (g) were analyzed by QRT-PCR $(n=5) .{ }^{*} P<0.05,{ }^{* \star} P<0.01,{ }^{* \star *} P<0.001$ versus untreated Ment ${ }^{\text {tlox }}$. (k and I) Fluorescent micrographs of dual calcein labeling (k) and its quantitative analysis of bone formation rate per bone surface (BFR/BS) (I) by dynamic histomorphometry in femurs of 12-week-old female Men $1^{\text {flox }}$ and Ment ${ }^{\text {Runx } 2 \text { Cre }}$ mice $(n=4$ or 5$)$. ( $\mathbf{m})$ Determination of the serum PINP level (a bone formation marker) in 12-week-old female Men $1^{\text {flox }}$ and Men $1^{\text {Runx } 2 \text { Cre }}$ mice $(n=5)$. Data are represented as mean \pm S.E.M. 
crossing Men1 $1^{\text {tm1.1Zqw }}$ (hereafter designated Men1 $1^{\text {flox }}$ ) mice ${ }^{13}$ to $\mathrm{Tg}$ (Runx2-icre)1Jtuc (hereafter designated Runx2Cre) mice ${ }^{14}$ to obtain Men $1^{\text {Runx2Cre }}$ mice. Immunohistochemistry of femurs indicated a decreased menin expression in osteoblasts and osteocytes of Men1 $1^{\text {Runx2Cre }}$ mice (Supplementary Figure S1a). Successful recombination was additionally confirmed by crossing a RosamT/mG (Tomato/ EGFP) reporter mouse ${ }^{15}$ to Runx2Cre mice and demonstrating cre-loxP recombination by GFP-positive osteoblasts and osteocytes (Supplementary Figure S1b). Men $1^{\text {Runx2Cre }}$ mice were viable without gross alterations of the skeleton (Supplementary Figure S1c) and were born slightly below the Mendelian ratio (Supplementary Table S1).

Strikingly, micro computed tomography (micro $\mathrm{CT}$ ) revealed a strong bone loss in the distal femur of female Men $1^{\text {Runx2Cre }}$ mice (Figures 1a-e). This bone loss was observed in 4-weekold mice and persisted upon aging (Figures $1 \mathrm{~b}-\mathrm{e}$ ). Similarly, bone loss was observed in male Men1 $1^{\text {Runx2Cre }}$ mice (Supplementary Table S2), resembling osteoporosis. Although trabecular thickness remained unaltered, the strong decrease of trabecular bone mass in Men $1^{\text {Runx2Cre }}$ mice was found to be caused by a low number of trabeculae and an increased trabecular separation (Figures $1 \mathrm{~b}-\mathrm{e}$ and Supplementary Table S2). Notably, cortical bone thickness in the femur was unchanged (Supplementary Table S3). Histomorphometry and micro CT analysis of tibiae and vertebrae confirmed the trabecular bone loss (Supplementary Figures S1d-h and Supplementary Table S2). Next, we analyzed whether the osteoporotic phenotype was specifically dependent on the elimination of Men1 in the osteoblast lineage.

First, we corroborated that the observed bone loss strictly depended on elimination of Men1 in the osteoblast lineage. Mice with a disruption of Men1 in the myeloid lineage (Lyz2 $^{\text {tm1 } 1 \text { (cre)lfo }}{ }^{16}$ hereafter designated Men $1^{\text {LysMCre }}$ mice) including osteoclasts (Supplementary Figures S2a and b) did not display any alterations in trabecular bone volume, trabecular thickness, numbers, and separation (Supplementary Figures S2c-f). In contrast, eliminating Men1 in the early differentiated osteoblast lineage using another osteoblast-specific cre-line, Tg(Sp7-tTA,tetO-EGFP/ cre)1Amc (hereafter designated OsxCre) mice, ${ }^{17}$ reproduced the severe osteoporosis as observed in Men $1^{\text {Runx2Cre }}$ mice. The resulting Men $1^{\text {OsxCre }}$ mice also displayed a strong decrease of trabecular bone volume and trabecular numbers and an increased trabecular separation, whereas trabecular thickness was only slightly affected (Supplementary Table S2).

Second, we asked whether loss of Men1 influenced the expression of osteoblast marker genes and osteoblast numbers in vivo. The mRNA expression of osteoblast marker genes such as Alpl, Col1a1, Runx2, and Bglap was similar in the calvarial bone of Men $1^{\text {Runx2Cre }}$ mice compared with control $\left(\right.$ Men $1^{\text {flox }}$ ) (Supplementary Figures S3a-d). In line with this, dynamic histomorphometry of vertebrae did not detect significant changes of osteoblast numbers and osteoblast surface (Supplementary Figures S3e and f). Osteocyte number was significantly higher in Men1 $1^{\text {Runx2Cre }}$ mice (Supplementary Figure S3g).

Furthermore, we asked whether Men1 deficiency could influence osteoblast differentiation in vitro as suggested in previous studies. ${ }^{7,12}$ To eliminate Men1 efficiently in osteoblast progenitor cells, we cultivated mesenchymal progenitor cells and primary calvarial osteoblasts derived from Men $1^{\text {flox }}$ mice crossed to a mouse line with ubiquitous expression of a Cre-ERT2 fusion protein (Gt(ROSA)26Sor ${ }^{\text {tm9(Cre/ESR1)Arte, }}$ hereafter designated gtRosaCreERT2), allowing a dramatic decrease in Men1 expression upon tamoxifen treatment (Supplementary Figures S3o, p, and s). Disruption of Men1 in vitro did not affect the growth of calvarial osteoblasts (Supplementary Figure S3h). Intriguingly, the differentiation of Men1-deficient calvarial osteoblasts and mesenchymal progenitor cells was unaffected and the cells responded normally to BMP2-induced differentiation as indicated by histochemical staining (Figures 1f, g and Supplementary Figures S3i-I) and marker gene expression (Figures $1 \mathrm{~h}-\mathrm{j}$, Supplementary Figures S3m and n). In accordance with these results, no major differences were observed in the BMP2-induced phosphorylation of Smad1/5/8 (Supplementary Figure S3p) and induction of the BMP2-Smad target genes Id1 and DIx5 (Supplementary Figures $\mathrm{S} 3 \mathrm{q}$ and $\mathrm{r}$ ) between control and Men1-deficient cells.

As it was previously reported that inhibiting Men1 expression with antisense oligonucleotides could affect TGF $\beta$ signaling, ${ }^{11}$ we also analyzed the TGF $\beta$-induced phosphorylation of Smad3 as well as the expression of TGF $\beta$-induced genes in the absence of Men1 in osteoblasts. No significant difference was found at the level of Smad3 phosphorylation, nor at expression levels of the TGF $\beta$ target genes Serpine and Skil in the absence of Men1 (Supplementary Figures S3s-u).

In accordance with the unaltered osteoblast differentiation, there was no difference in the bone formation rate in femurs (Figures $1 \mathrm{k}$ and $\mathrm{I}$ ) and vertebrae (Supplementary Figures S3v and $\mathrm{w}$ ) of 12-week-old Ment $1^{\text {flox }}$ and Ment $1^{\text {Runx2Cre mice. }}$ Correspondingly, the serum PINP level was not changed in Men $1^{\text {Runx2Cre }}$ mice (Figure $1 \mathrm{~m}$ ). However, the bone formation rate in 12-month-old Men1 ${ }^{\text {Runx2Cre }}$ mice was significantly reduced (Supplementary Figures S3x and y). This corroborates the finding of Kanazawa et al. ${ }^{7}$ who described impaired osteoblast function in 9- and 12-month-old mice in which Men1 was eliminated by an osteocalcin-cre.

In summary, despite a possible role for Men1 in bone formation during aging, no drastic changes of osteoblast marker gene expression, osteoblast number, and osteoblast function were observed in young and middle-aged mice lacking Men1 in the osteoblast lineage.

Men1 deficiency in the osteoblast lineage, specifically in osteocytes, leads to an enhanced osteoclastogenesis. We observed a strong increase of osteoclast numbers and surface in femurs (Figures $2 a$ and b) and vertebrae (Supplementary Figures S4a and b). These increases were also seen in calvaria (Supplementary Figures S4c-e) and were associated with a high porosity (Supplementary Figure S4f). Bone resorption as determined by serum C-terminal telopeptide (CTX) level was increased in 12-week-old Men1 ${ }^{\text {Runx2Cre }}$ mice (Figure 2c). To test whether Men1-deficient osteoblasts could cause greater osteoclastogenesis, we performed osteoblast-osteoclast co-culture by using control or Men1-deficient osteoblasts and wild-type osteoclast precursors. Surprisingly, the Men1-deficient 

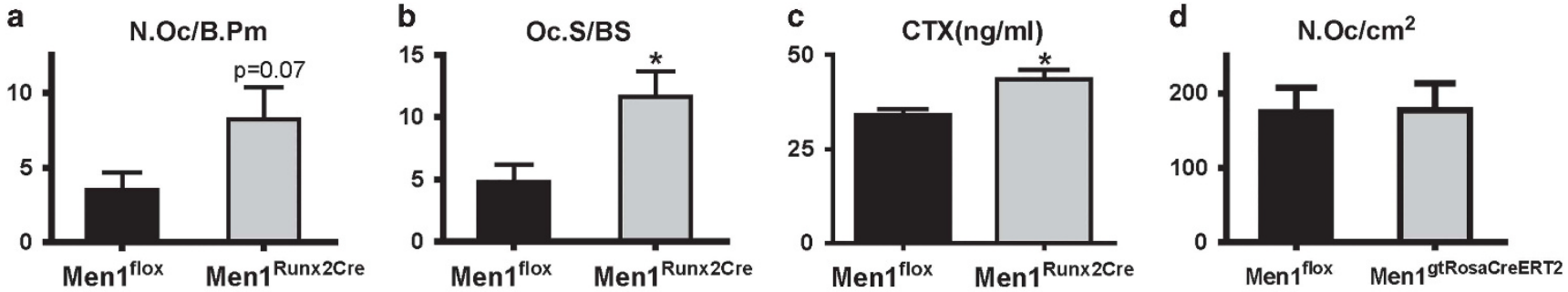

e

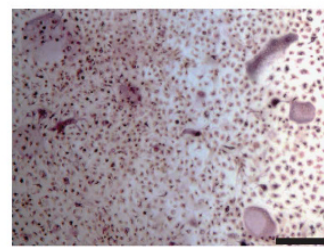

Men1 $1^{\text {flox }}$

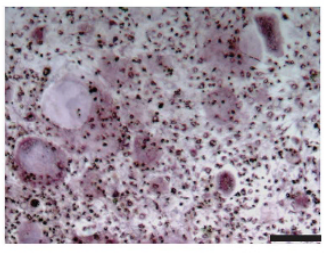

Men1 ${ }^{\text {gtRosaCreERT2 }}$
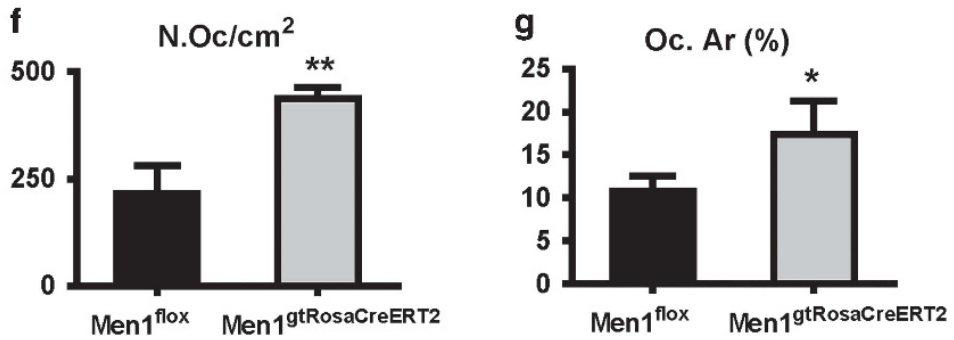

Figure 2 Bone resorption is enhanced in Men1 ${ }^{\text {Runx2Cre }}$ mice, caused by Men1 deficiency in osteocytes. (a and b) Osteoclast numbers per bone perimeter (N.Oc/B.Pm) (a) and osteoclast surface per bone surface (Oc.S/BS) (b) in sections of femoral trabecular bone were measured by histomorphometry $(n=4$ or 5$)$. (c) Determination of resorption by assessment of the serum level of the biomarker CTX in 12-week-old female Men $1^{\text {flox }}$ and Men $1^{\text {Runx2Cre }}$ mice $(n=5)$. (d) Number of multinucleated TRAP-positive osteoclasts was counted from primary co-cultures of wild-type BMCs with Men $1^{\text {flox }}$ or Men $1^{\text {gtRosaCreERT2 }}$ primary osteoblasts $(n=3)$. (e-g) Osteoclastogenesis of primary co-cultures of wild-type BMCs with Men $1^{\text {flox }}$ or Men $1^{\text {gtRosaCreERT2 }}$ primary osteocytes was visualized by TRAP staining (e). Number of multinucleated TRAP-positive cells (f) and their area (g) were determined $(n=3)$. ${ }^{*} P<0.05,{ }^{*} P<0.01$. Data are represented as mean \pm S.E.M. Scale bar: $25 \mu \mathrm{m}$

osteoblasts could not trigger higher osteoclast numbers (Figure 2d).

Osteocytes were recently established as major producers of RANKL in bone and therefore important regulators of osteoclastogenesis. ${ }^{1,2}$ As deletion of Men1 by the cre-loxP system in osteoblasts also affects Men1 expression in osteocytes (Supplementary Figures S1a and b), we further tested the capacity of Men1-deficient osteocytes to stimulate osteoclast formation ex vivo. To this end, we isolated primary osteocyte-enriched fractions from Men $1^{\text {gtRosaCreERT2 }}$ mice. These cells expressed higher Dmp1 and lower Alpl levels when compared with primary osteoblasts, confirming the efficacy of the isolation of osteocytes (Supplementary Figures $\mathrm{S} 5 \mathrm{a}$ and $\mathrm{b})$. Following 4-hydroxytamoxifen (4-OHT) treatment to eliminate the Men1 gene (Supplementary Figure S5c), the osteocyte-enriched fraction was co-cultivated with wild-type bone marrow cells (BMCs) containing osteoclast progenitor cells. As a result, osteoclast number and osteoclast area were significantly higher in the co-culture containing Men1-deficient osteocytes (Figures 2e-g).

Men1 deficiency in osteocytes leads to an enhanced osteoclastogenesis in vivo. The in vitro co-culture experiments suggested that the increased osteoclast number in the mice lacking Men1 in the osteoblastic lineage was conferred by Men1-deficient osteocytes rather than osteoblasts. To confirm this hypothesis in vivo, we generated mice lacking Men1 in osteocytes, but not in early differentiated osteoblasts, by crossing Men $1^{\text {flox }}$ mice to Tg(Dmp1-cre)1Jqfe (hereafter designated Dmp1Cre) mice $^{18}$ to obtain Men $1^{\text {Dmp1Cre }}$ mice. Loss of menin in osteocytes in Men $1^{\text {Dmp1Cre }}$ mice was confirmed by immunohistochemistry (Supplementary Figure S5d) and by crossing Men $1^{\text {Dmp1Cre }}$ mice to Rosa ${ }^{\mathrm{mT} / \mathrm{mG}}$ reporter mice that displayed a strongly diminished Men1 expression in GFP-positive cells (Figure 3a and Supplementary Figure S5e). As Dmp1 is also expressed in mature osteoblasts, we stained osteocalcin as a mature osteoblast marker in Men $1^{\mathrm{Dmp} 1 \mathrm{Cre}} ;$ Rosa $^{\mathrm{mT} / \mathrm{mG}}$ reporter mice (Supplementary Figure S5f) to determine the degree of recombination in these cells. We found that $29 \pm 9 \%$ of osteocalcin-stained mature osteoblasts were EGFP positive, indicating that a minor fraction of the late-stage differentiated osteoblasts is also mutant in addition to osteocytes in Men $1^{\mathrm{Dmp} 1 \mathrm{Cre}} ; \mathrm{Rosa}^{\mathrm{mT} / \mathrm{mG}}$ mice. Micro CT analysis revealed a severe osteoporosis in the distal femur of adult Men $1^{\text {Dmp1Cre }}$ mice (Figures $\left.3 b-f\right)$. The decrease of trabecular bone mass in Men1 $1^{\text {Dmp1Cre }}$ mice was because of a low number of trabeculae and an increased trabecular separation. Trabecular thickness was again unchanged. Osteoblast number, osteoblast surface, and osteocyte number were not altered in Men $1^{\text {Dmp1Cre }}$ mice (Figures $3 \mathrm{~g}-\mathrm{i}$ ). Accordingly, the bone formation rate and serum PINP level were not different between Men $1^{\text {flox }}$ mice and Men $1^{\text {Dmp1Cre }}$ mice (Figures 3j-I). Osteoclast number, osteoclast surface, and CTX level were significantly elevated in Men1 ${ }^{\text {Dmp1Cre }}$ mice (Figures $3 \mathrm{~m}-0$ ), whereas RANKL and OPG levels were not changed (Supplementary Figures S5g and h). Thus, a similar bone phenotype was observed in Men $1^{\mathrm{Dmp} 1 \mathrm{Cre}}$ mice as shown in Men $1^{\text {Runx2Cre }}$ and Men $1^{\text {OsxCre }}$ mice. Together with the observed increase of osteoclastogenesis by osteocytes, these observations strongly suggest that osteoporosis by loss of Men1 is executed by osteocytes in these three different conditional knockout mouse strains.

Menin suppresses CXCL10 to regulate osteocyte-osteoclast crosstalk. We could not detect differences in Tnfsf11 (RANKL) and Tnfrsf11b (OPG) expression in the bone of Men $1^{\text {Dmp1Cre }}$ mice (Supplementary Figures S6a and b). We 
a

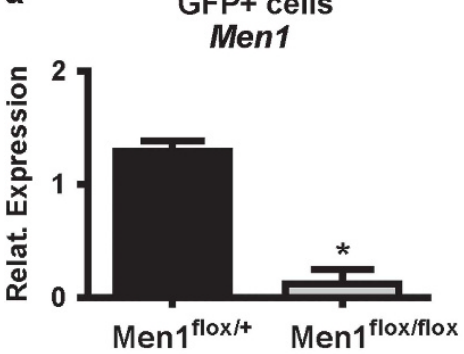

d

d Tb.Th. $(\mu \mathrm{m})$

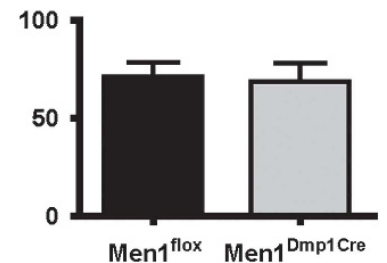

f

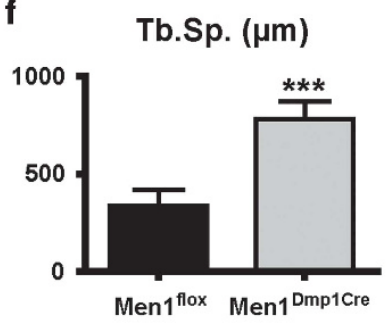

h

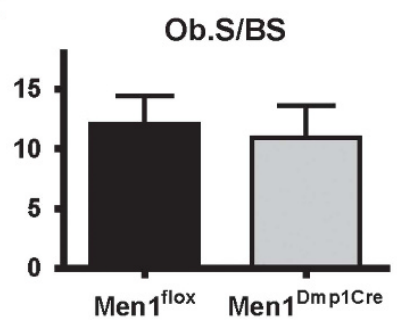

I

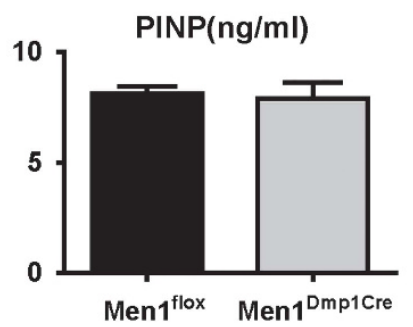

b

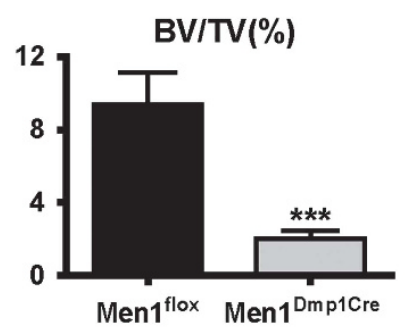

e

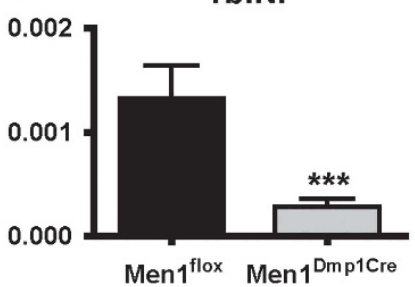

9

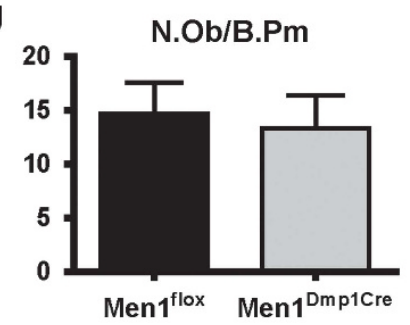

i
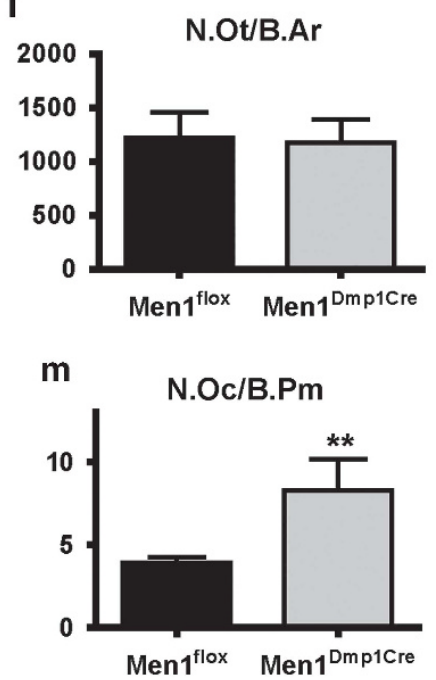

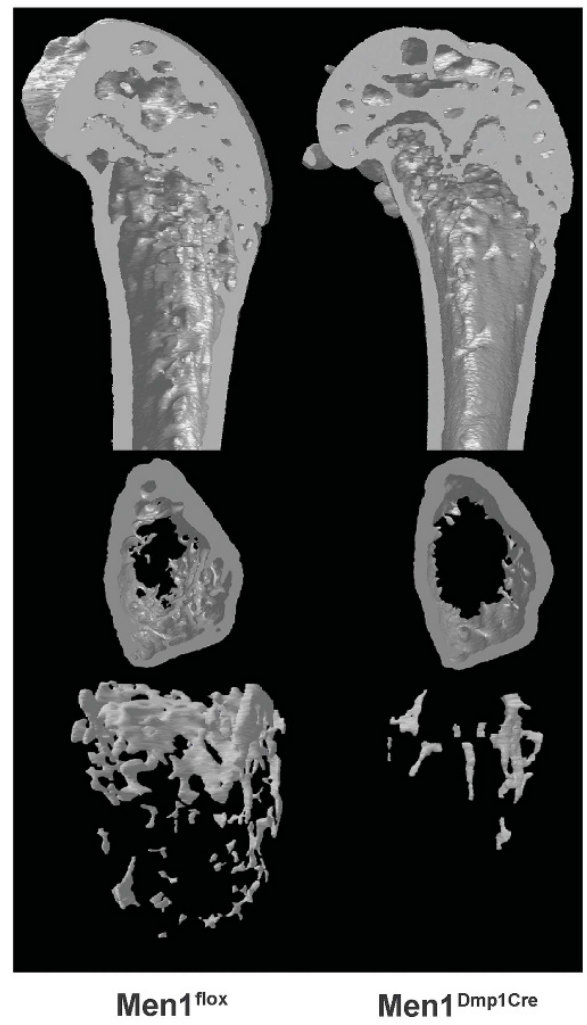

j
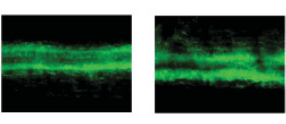

Men $1^{\text {flox }}$ Men1 $1^{\text {Dmp1cre }}$

n

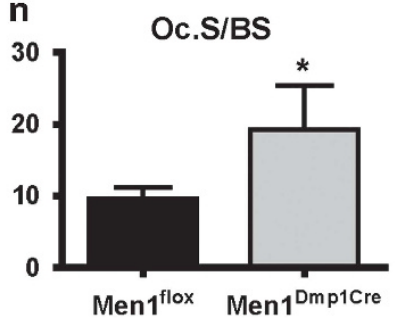

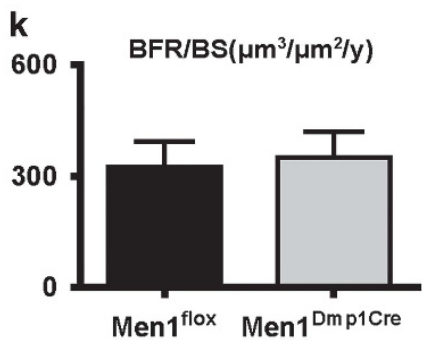

- CTX (ng/ml)

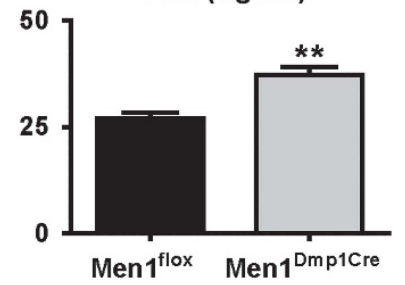

Figure 3 Men1 deficiency in osteocytes leads to an enhanced osteoclastogenesis in vivo. (a) Real-time PCR analysis of Men1 mRNA expression in FACS-sorted GFPpositive cells from femoral bone of Men1 ${ }^{\text {Dmp1Cre }}$ mice crossed to Rosa ${ }^{\mathrm{mT} / \mathrm{mG}}$ mice in animals heterozygous and homozygous for Mentflox. (b-f) Micro CT reconstruction (c) of femurs from 12-week-old female Men $1^{\text {flox }}$ and Men1 $1^{\text {Dmp1Cre }}$ mice. Cancellous parameters such as BV/TV (b), Tb.Th. (d), Tb.N. (e), and Tb.Sp. (f) were measured from distal femurs of 12-week-old female Men $1^{\text {flox }}$ and Men $1^{\text {Dmp1Cre }}$ mice by micro CT ( $n=4$ or 5). (g-i) Osteoblast number per bone perimeter (N.Ob/B.Pm) (g), osteoblast surface per bone surface (Ob.S/BS) (h), and osteocyte number per bone area (N.Ot/B.Ar) (i) in trabecular bone of distal femoral sections from 12-week-old female Men $1^{\text {flox }}$ and $\mathrm{Men} 1^{\mathrm{Dmp}}$ (Cre mice were measured by histomorphometry $(n=4$ or 5 ). ( $\mathbf{j}$ and $\mathbf{k}$ ) Fluorescent micrographs of dual calcein labeling (j) and its quantitative analysis of BFR/BS (k) in femoral sections of 12-week-old female Men $1^{\text {flox }}$ and Men $1^{\text {Dmpicre }}$ mice $(n=4$ or 5$)$. (I) Determination of the serum PINP level in 12-week-old female Men $1^{\text {flox }}$ and Men $1^{\text {Dmp1Cre }}$ mice $(n=5)$. ( $\mathbf{m}$ and $\mathbf{n})$ N.Oc/B.Pm $(\mathbf{m})$ and Oc.S/BS $(\mathbf{n})$ in trabecular bone of distal femoral sections from 12-week-old female Men $1^{\text {flox }}$ and Ment $1^{\text {Dmp1Cre }}$ mice were measured by histomorphometry $\left(n=4\right.$ or 5). (o) Determination of resorption from the serum CTX level in 12-week-old female Men $1^{\text {flox }}$ and Men $1^{\mathrm{Dmp} 1 \mathrm{Cre}}$ mice $(n=5)$. ${ }^{\star} P<0.05,{ }^{\star *} P<0.01$, ${ }^{* * *} P<0.001$. Data are represented as mean \pm S.E.M. 


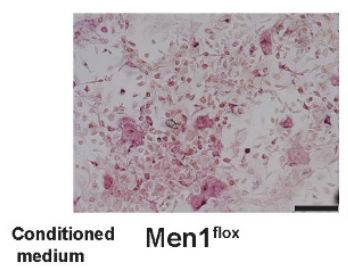

d

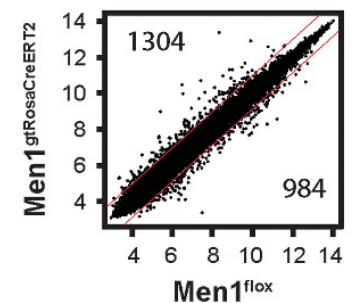

b

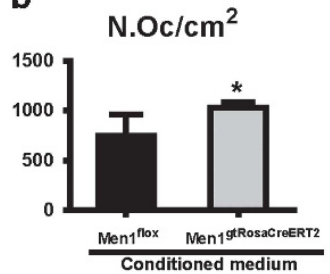

c

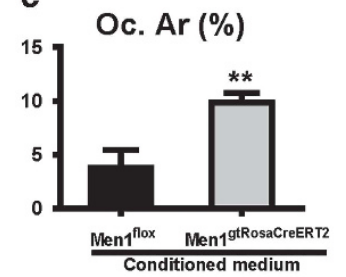

e

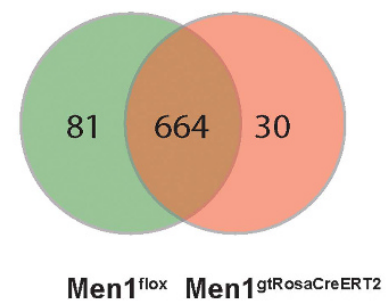

f
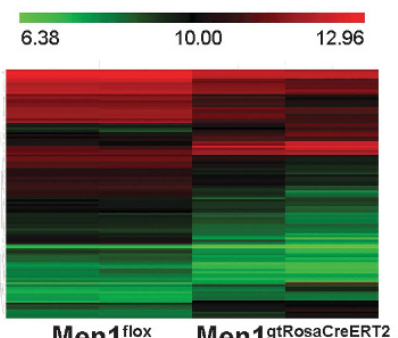
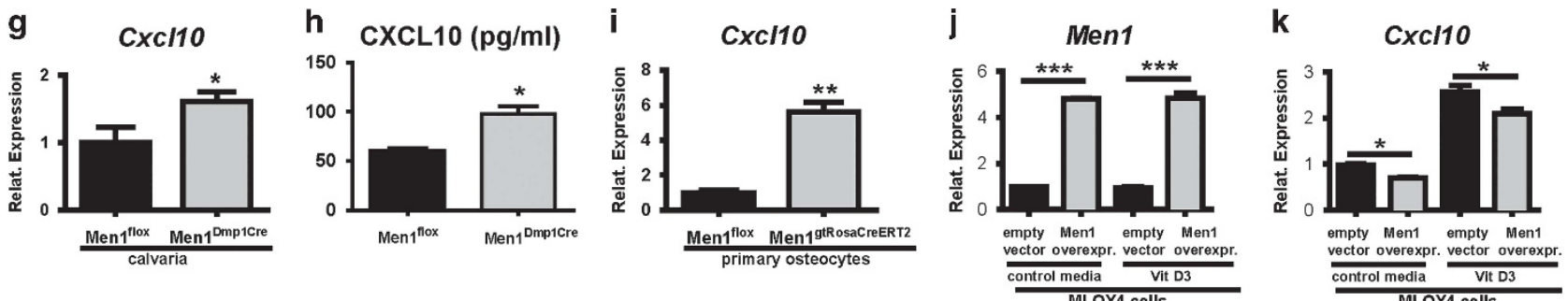

Figure 4 Men1 deficiency in osteocytes leads to an enhanced osteoclastogenesis via increased Cxcl10 expression. (a-c) Osteoclastogenesis was visualized by TRAP staining of co-cultures of primary wild-type osteocytes and BMCs supplemented with conditioned medium from 4-OHT-pretreated Men flox $^{\text {or }}$ Men $1^{\text {gtRosaCreERT2 }}$ osteocyte cultures (a). Number of multinucleated TRAP-positive cells (b) and their area (c) were determined $(n=3)$. (d-f) Microarray analysis of Men1-deficient enriched osteocyte fractions. (d) Scatter plot of normalized expression levels of control (Ment $1^{\text {flox }}, \mathrm{x}$ axis) and Men1-deficient (Men $1^{\text {gtRosaCreERT2 }}$, y axis) enriched osteocyte fractions. Probe sets outside of the two diagonal lines represent 1.4-fold upregulated or 0.7-fold downregulated genes in Men1-deficient cells compared with Men $1^{\text {flox }}$ control cells. (e) Venn diagram of Men1-regulated genes annotated as 'secreted' by DAVID. Numbers indicate genes highly expressed in Ment $1^{\text {flox }}$ cells (left), no change (middle), or highly expressed in Men $1^{\text {thiosaCreERT2 }}$ cells (right), respectively. (f) Heat map of normalized expression levels of control (WT, Men $1^{\text {flox }}$ ) and Men 1-deficient (KO, Men 1 $^{\text {tRosaCreERT2}}$ ) osteocytes. Secreted factors with high expression levels ( $>50 \%$ of the total probe signals) with fold change values ( $2^{\text {expression levels (KO-WT) }}$ of $>1.4$ (30 gene data sets) or $<0.7$ ( 81 gene data sets) were visualized in the heat map. (g) mRNA levels of Cxcl10 were determined from calvarial bone of 3-day-old Men $1^{\text {flox }}$ and Men1 ${ }^{\text {DmpiCre }}$ mice by QRT-PCR ( $n=4$ or 5). (h) CXCL10 levels in the supernatant after 5 days of organ culturing of calvaria from neonatal Men $1^{\text {Dmp1Cre }}$ mice were determined by ELISA $(n=3$ or 4$)$. (i) mRNA levels of Cxcl10 were determined by QRT-PCR in 4-OHT-pretreated enriched osteocyte fractions isolated from Men $1^{\text {flox }}$ and Men $1^{\text {gtRosaCreERT2 }}$ mice $(n=3)$. (j and $\left.\mathbf{k}\right)$ mRNA levels of Men1 (j) and Cxcl10 (k) were determined by QRT-PCR in Men1-overexpressing MLO-Y4 cells after treatment with control media or vitamin D3 $(n=3) .{ }^{*} P<0.05,{ }^{* *} P<0.01,{ }^{* * *} P<0.001$. Data are represented as mean \pm S.E.M. Scale bar: $25 \mu \mathrm{m}$

therefore tested whether other soluble factors derived from Men1-deficient osteocytes affected osteoclastogenesis. Transfer of conditioned medium from cultured Men1-deficient osteocyte-enriched fractions to wild-type osteocyte-enriched fractions/osteoclast co-cultures revealed a higher osteoclast formation than the transfer of medium from wild-type osteocyte-enriched fractions (Figures $4 a-c$ ). Furthermore, the conditioned medium from osteocyte-enriched fractions was added to wild-type osteoclast cultures. Again, the conditioned medium from Men1-deficient osteocyteenriched fractions triggered higher osteoclast formation than the medium from control osteocyte-enriched fractions (Supplementary Figures S6c-e).

Our findings from the transfer of conditioned medium suggested that the presence of soluble factors derived from osteocytes lacking Men1 influenced osteoclastogenesis. Subsequently, we performed a microarray analysis with the mRNA of osteocyte-enriched fractions (Figures 4d-f). In total, 2288 differentially expressed genes were found (Figure 4d). Of these, 775 genes (Figure 4e) were annotated as secreted factors analyzed by DAVID bioinformatics resources. ${ }^{19}$ Of the 775 genes, 81 were upregulated in wild-type osteocytes and 30 were more highly expressed in Men1-deficient osteocyteenriched fractions (Figure 4f, e and Supplementary Table S4). Among these 111 genes, 12 were related to osteoclastogenesis based on the literature. All of the 12 osteoclastogenesisassociated genes were subsequently analyzed for their expression in entire calvarial bone of Men $1^{\mathrm{Dmp} 1 \mathrm{Cre}}$ mice by QRT-PCR (Figure $4 \mathrm{~g}$ and Supplementary Figures S6f-p). Of these genes, C-X-C motif chemokine 10 (CXCL10), a previously described osteoclastogenesis-inducing factor, ${ }^{20}$ showed the most strikingly elevated expression in entire calvarial bone of Men $1^{\text {Dmp1Cre }}$ mice (Figure $4 \mathrm{~g}$ ). We also detected enhanced CXCL10 secretion in supernatant medium of organ cultures of calvarial bones (Figure 4h). Real-time PCR confirmed a significantly higher $\mathrm{CxCl} 10$ expression in Men1- 


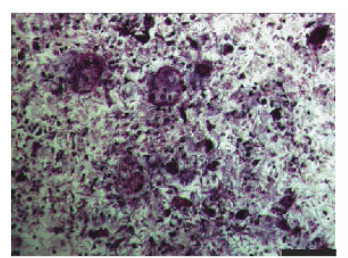

$\lg G$

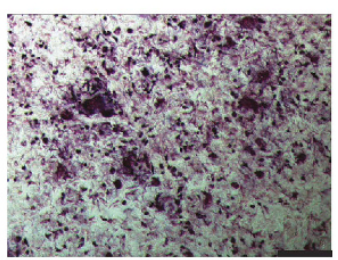

Cxcl10 antibody b

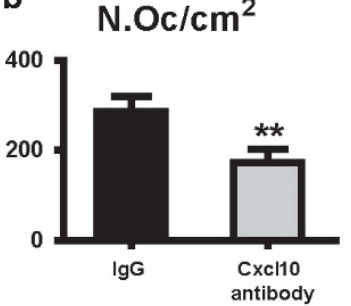

C

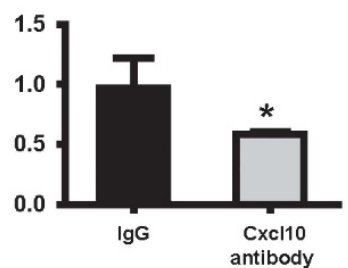

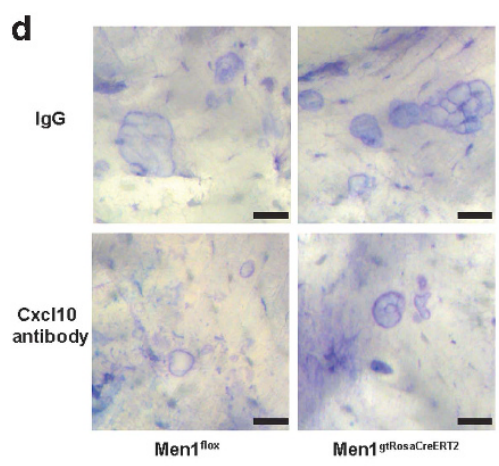

d

g

$\lg G$

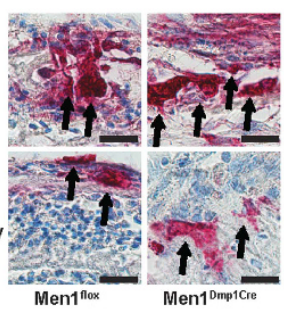

k

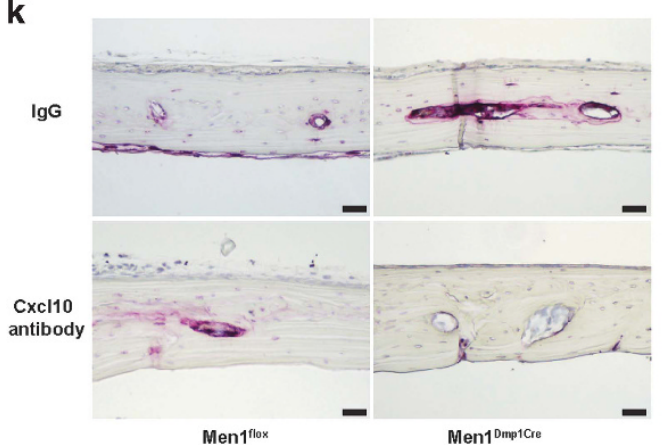

e

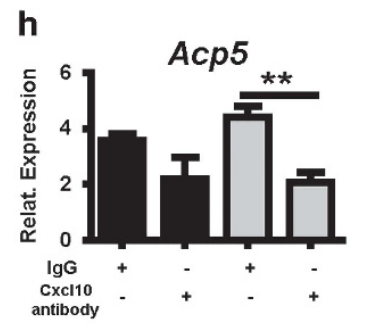

i

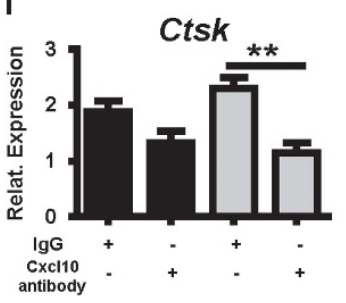

I

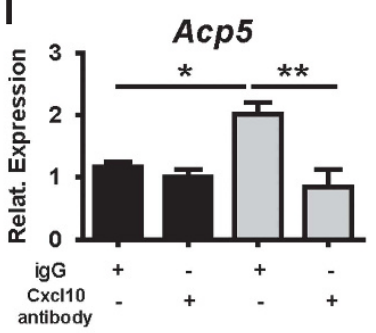

j

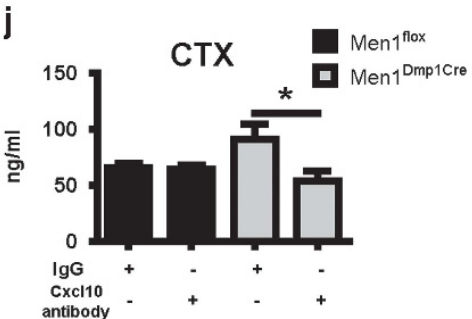

m

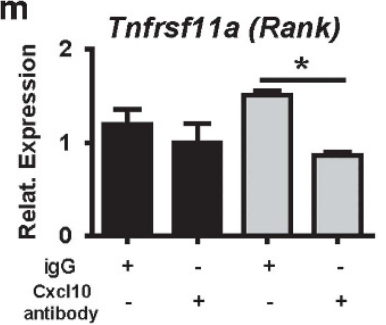

Figure 5 CXCL10-neutralizing antibody rescues enhanced osteoclastogenesis. (a-c) CXCL10-neutralizing antibody (20 ng/ml) or lgG was added to co-cultures of wild-type

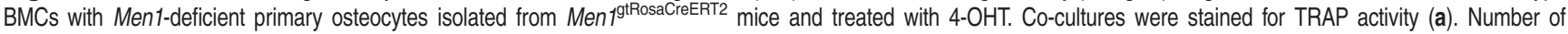
multinucleated TRAP-positive cells $(\mathbf{b})$ and their area (c) were determined $(n=3)$. (d-f) CXCL10-neutralizing antibody (20 ng/ml) or lgG was added to co-cultures of wild-type

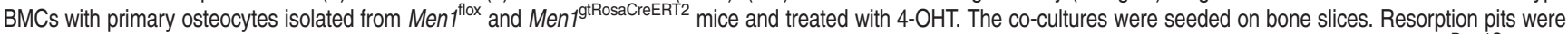
stained with toluidine blue (d). Pit area (e) and CTX levels in the supernatant (f) were determined $(n=3)$. (g-j) Organ cultures of calvaria from neonatal Men $1^{D m p i C r e}$ mice were treated with lgG or CXCL10-neutralizing antibody. TRAP staining was performed and osteoclasts were visualized (arrows) (g). Acp5 (h) and Ctsk (i) mRNA levels were determined by QRT-PCR. CTX levels in the supernatant (j) were determined by ELISA ( $n=3$ or 4$)$. ( $k-\mathbf{m})$ lgG or CXCL10-neutralizing antibody was subcutaneously injected over the calvaria of 12-week-old female Ment $1^{\text {flox }}$ and Men $1^{\text {Dmp1Cre }}$ mice. TRAP staining was performed (k). Acp5 (I) and Tnfrsf11a (m) mRNA levels were determined by QRT-PCR $(n=4) .{ }^{*} P<0.05,{ }^{* *} P<0.01,{ }^{* * *} P<0.001$. Data are represented as mean \pm S.E.M. Scale bar: $25 \mu \mathrm{m}$

deficient primary osteocytes (Figure 4i). Similar results were found from calvaria of Men $1^{\text {Runx2Cre }}$ mice (Supplementary Figure S6q). Contra to the increased $\mathrm{CxCl10}$ expression in Men1-deficient osteocyte-enriched fractions, Men1 overexpression in the MLO-Y4 osteocyte cell line (Figure 4j) significantly diminished basal and 1,25-dihydroxyvitamin
D3-induced levels of $\mathrm{Cxc} / 10$ expression (Figure 4k). These data indicate a specific role for Men1 in osteocytes in the regulation of $\mathrm{CxCl10}$ expression. Furthermore, CXCL10neutralizing antibodies significantly reduced osteoclastogenesis in wild-type BMCs co-cultured with Men1-deficient osteocyte-enriched fractions (Figures $5 \mathrm{a}-\mathrm{c}$ ). Of note, these 
neutralizing antibodies did not affect wild-type osteocyteenriched fractions co-cultured with BMCs (Supplementary Figures S6r and s). In order to assess the resorption ability in vitro, the co-cultures were performed on bone slices. Co-cultures with Men1-deficient osteocyte-enriched fractions displayed a clear tendency of higher resorption pit area and significantly increased CTX release that were significantly reduced by the CXCL10-neutralizing antibodies (Figures 5d-f).

We verified these findings in organ cultures of calvaria derived from Men $1^{\text {Dmp1Cre }}$ mice. TRAP staining, osteoclast marker expression, and the level of resorption marker CTX in the supernatant were all decreased upon CXCL10-neutralizing antibody treatment (Figures $5 \mathrm{~g}-\mathrm{j}$ ), indicating that CXCL10 mediates increased osteoclastogenesis and resorption in intact bones.

Furthermore, CXCL10-neutralizing antibodies were subcutaneously injected over the calvaria of Men $1^{\text {flox }}$ and Men $1^{\text {Dmp1Cre }}$ mice. Osteoclast indicating TRAP staining was reduced in CXCL10-neutralizing antibody-treated Men $1^{\text {Dmp1Cre }}$ mice (Figure $5 \mathrm{k}$ ). Osteoclast marker expression was also reduced in the calvaria of these mice (Figures $5 \mathrm{l}$ and $\mathrm{m}$ ). Thus, these data demonstrate the reduction of enhanced osteoclastogenesis in Men $1^{\mathrm{Dmp} 1 \mathrm{Cre}}$ mice by CXCL10-neutralizing antibodies in vivo.

In summary, Men1 deficiency specifically in osteocytes increases $\mathrm{Cxcl} 10$ expression and enhances osteoclastogenesis in vitro and in vivo that contributes to the osteoporotic phenotype observed in mice with a disruption of Men1 in osteoblast-derived osteocytes $\left(\right.$ Men $1^{\text {Runx2Cre }}$, Men1 ${ }^{\text {OsxCre }}$, and Men $1^{\text {Dmp1Cre }}$ mice).

\section{Discussion}

In this study, we uncover the role of Men1 in maintaining bone integrity via osteocyte-osteoclast crosstalk. Men1 deficiency in the osteoblast lineage does not affect bone formation, whereby Men1 deficiency in osteocytes promoted osteoclastogenesis by enhancing the production of soluble factors, such as CXCL10, causing osteoporosis in young or middleaged mice.

In agreement with a previous study, ${ }^{7}$ we observed a decrease of bone formation in aged 12-month-old female mice. Strikingly, in middle-aged mice, we unequivocally show that selective Men1 deletion in the osteoblastic lineage in three distinct conditional Men1 mutant mouse lines does not alter bone formation, as indicated by the bone formation rate in femurs and vertebrae as well as systemic PINP levels. Our conclusion is supported by the ex vivo analysis of osteoblast differentiation in the absence of Men1 in uncommitted mesenchymal stromal cells and neonatal calvarial osteoblasts. In primary uncommitted mesenchymal stromal cells from 6- to 8-week-old mice and neonatal calvarial osteoblasts from 3- to 5-day-old newborn mice with an efficient deletion of Men1 using an inducible cre-loxP system, we did not detect any differences in osteoblast proliferation and their differentiation potential. This is in contrast to previous studies by Kanazawa et al. ${ }^{7}$ who described a decreased osteoblast differentiation of primary calvarial cells derived from 6-monthold Men1flox-osteocalcin-cre mice. In addition, previous studies suggested a decrease of BMP2-Smad1/5/8 signaling and an increase of TGF $\beta$ signaling upon abrogation of Men1. ${ }^{7,11,12}$ In osteoblast progenitor cells with a complete elimination of Men1, no major difference was evident in either Smad1/5/8 phosphorylation or Smad3 phosphorylation upon BMP2 and TGF $\beta$ stimulation, respectively. Furthermore, the response of BMP2 and TGF $\beta$ target genes was unaltered and, importantly, the effect on osteoblast differentiation was unchanged in calvarial and mesenchymal stromal cells. These discrepancies may be explained in part by the fact that antisense oligonucleotides were used in the in vitro studies, ${ }^{7,11,12}$ a technology associated with many undefined off-target effects. The primary cells analyzed by Kanazawa et al. ${ }^{7}$ were derived from aged calvaria and we cannot exclude the possibility that such old cells devoid of Men1 display a different response. In summary, our findings support the lack of alterations in osteoblast numbers and the bone formation rate seen in 3-month-old Men $1^{\text {Runx2Cre }}$ mice in vivo.

In this study, we show a novel role for Men1 in osteocytes governing bone resorption. Osteocytes were recently identified as the major source of RANKL in adult mice that is essential for bone resorption. ${ }^{1,2}$ We found in the absence of Men1 in osteoblasts an increased osteocyte number that was also observed in Men 1flox-osteocalcin-cre mice. ${ }^{7}$ However, in Men $1^{\text {Dmp1Cre }}$ mice, osteocyte numbers were unaltered, indicating that Men1 within osteocytes does not affect the abundance of these cells. Nonetheless, in Men $1^{\mathrm{Dmp} 1 \mathrm{Cre}}$ mice, despite unaltered RANKL expression, we also observed enhanced osteoclast numbers and enhanced resorption based on increased CTX levels in the serum and co-cultures of Men1-deficient osteocyte-enriched fractions with osteoclasts. Given that bone mass is already substantially reduced, the elevated levels of CTX indicate a massive enhanced resorption activity in the remaining bone. This was not due to an intrinsic effect of Men1 in osteoclasts, as there is no recombination in osteoclasts in Runx2Cre, ${ }^{14}$ OsxCre, ${ }^{17}$ and Dmp1Cre mice. ${ }^{18}$ Accordingly, Men $1^{\text {LysMCre }}$ mice exhibiting a loss of Men1 in myeloid cells displayed a normal bone phenotype, confirming that bone mass is not affected by Men1 in osteoclasts.

However, because of the fact that Dmp1Cre recombination occurs in osteocytes as well as in $29 \pm 9 \%$ of late-stage osteoblast populations $^{1}$ (Supplementary Figure S5f), we cannot fully exclude a possible contribution of Men1-deficient osteoblasts to the enhanced osteoclastogenesis. However, the failure of Men1-deficient osteoblasts, in contrast to Men1deficient osteocyte-enriched fractions, in enhancing osteoclastogenesis and resorptive activity in co-cultures favors a dominant role of Men1 in osteocytes in suppressing osteoclastogenesis in vivo.

This is further supported by enhanced release of several soluble factors from Men1-deficient osteocytes as being a causative determinant for enhanced osteoclastogenesis. For example, conditioned medium derived from Men1-deficient osteocyte-enriched fractions displayed an enhanced osteoclastogenic potential, revealing for the first time the role of osteocyte-secreted factors in causing greater osteoclastogenesis. The annotation toward secreted factors from an RNA expression profile defined a potential secretome containing candidate genes important for osteocyte-osteoclast crosstalk 
beyond the regulation of RANKL. Among those identified, CXCL10 was found as most strongly induced in entire bone because of a dramatic upregulation found in enriched osteocyte fractions. CXCL10 has been reported to stimulate osteoclast differentiation. ${ }^{20}$ Furthermore, the menininteracting partner MLL regulates Cxc/10 expression in macrophages and this effect can be abrogated using an MLL-menin inhibitor. ${ }^{21}$ We found a clear negative regulation of $\mathrm{CxCl10}$ expression by Men1, as it was elevated in primary osteocytes and bones from Men1 $1^{\text {Dmp1Cre }}$ mice and Men $1^{\text {Runx2Cre }}$ mice and reduced in Men1-overexpressing MLO-Y4 osteocyte cells. The functional relevance of enhanced $\mathrm{Cxc} / 10$ expression was demonstrated by CXCL10-neutralizing antibody treatment of (1) co-cultures, (2) calvarial organ cultures, and (3) mice that resulted in decreased osteoclast formation and resorption as indicated by decreased TRAP staining, osteoclast marker gene expression, and CTX levels. Our data underscore a crucial role of menin in the negative regulation of $\mathrm{Cxc} / 10$ transcription that could be, at least partially, the molecular basis of the enhanced osteoclastogenesis triggered by Men1 disruption in osteocytes. Given that MDX-1100, an antibody against CXCL10, is in clinical trial phase II for treatment of arthritic patients, ${ }^{22}$ this may prove to be an attractive agent to improve bone quality.

Despite the general role of Men1 in bone integrity, Men1 conditional knockout mice allow the elucidation of the intrinsic consequences of loss of MEN1 that might occur in patients suffering from MEN1. The osteopenic phenotype of these patients may be masked by their PTH oversecretion. Intriguingly, following parathyroidectomy, bone recovery is worse in MEN1 patients than in other hyperparathyroidism conditions. ${ }^{23}$ Our findings will encourage future analyses on possible mutations in the osteoblastic lineage of MEN1 patients that will enable the PTH effects and osteocyte cellautonomic effects on bone integrity to be distinguished.

In conclusion, we have established a novel regulatory axis of osteocyte-osteoclast crosstalk regulated by the tumor suppressor gene Men1. Men1 acts in osteocytes to protect trabecular bone mass by modulation of osteoclast function. A Men1-regulated chemokine, CXCL10, was identified as an osteocyte-osteoclast crosstalk factor. Modulating Men1-regulated factors in osteocytes might serve as a novel molecular base to develop therapeutic strategies to treat osteoporosis.

\begin{abstract}
Materials and Methods
Mice. All mice were kept under standardized conditions with water and food ad libitum in specialized pathogen-free animal facilities at the University of UIm. Procedures for performing animal experiments were in accordance with and approved by the authorities for ethical permission (the Regierungspräsidium in Tübingen, Germany). Ment $1^{\text {Runx2Cre }}$, Men1 $1^{\text {sxCre }}$, Men $1^{\text {gtRosaCreERT2 }}$, Ment $1^{\text {LysMCre }}$,

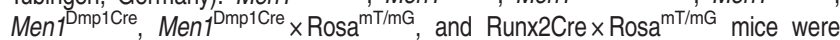
generated by intercrossing Men $f^{\text {tlox }}$ mice ${ }^{13}$ with $\mathrm{Tg}$ (Runx2-icre) 1 Jtuc mice, ${ }^{14} \mathrm{Tg}$ (Sp7-tTA, tetO-EGFP/cre)1Amc mice, ${ }^{17}$ C57BL/6-Gt(ROSA)26Sor ${ }^{\text {tm9(Cre/ESR1)Arte }}$ mice (Taconic Artemis, Köln, Germany), Lyz2 ${ }^{\text {th1(cre)lfo }}$ mice, ${ }^{16} \mathrm{Tg}$ (Dmp1-cre)1Jqfe mice, ${ }^{18}$ and Gt(ROSA)26Sortm4(ACTB-tdTomato,-EGFP)Luo/J mice, ${ }^{15}$, respectively
\end{abstract}

Micro computed tomography. Femurs, tibiae, and lumbar vertebrae were analyzed using a SkyScan 1174 compact micro CT or SkyScan 1176 in vivo micro CT (Bruker, Billerica, MA, USA) equipped with an X-ray tube working at 50-80 kV/100 $\mu \mathrm{A}$. Resolution was $6-9 \mu \mathrm{m}$, rotation step was set at $0.40^{\circ}$ or $1^{\circ}$, and a $0.5 \mathrm{~mm}$ aluminum filter was used. For reconstruction of femurs, the region of interest was defined $0.3 \mathrm{~mm}$ from the distal growth plate into the diaphysis spanning
$1.8 \mathrm{~mm}$. Trabecular bone volume/tissue volume (BV/TV), trabecular thickness (Tb.Th.), trabecular separation (Tb.Sp.), trabecular number (Tb.N.), cortical thickness (Cs.Th.), cortical closed porosity, and cortical bone perimeter (B.Pm.) were determined according to the guidelines issued by the ASBMR Histomorphometry Nomenclature Committee. ${ }^{24,25}$

Histomorphometry. Static and dynamic histomorphometry was performed on undecalcified and decalcified lumbar vertebral and femoral sections of mice receiving dual calcein (Sigma-Aldrich, St. Louis, MO, USA) i.p. injections, as described previously. ${ }^{25,26}$ Osteomeasure software was used for analysis (Osteometrics, Decatur, GA, USA).

Osteoblast differentiation from stromal and calvarial cells. Stromal cells were isolated from inguinal white adipose tissue of Men $1^{\text {gtRosaCreERT2 }}$ mice (6-8 weeks) by digestions for $30 \mathrm{~min}$ at $37^{\circ} \mathrm{C}$ in isolation buffer containing $100 \mathrm{mM}$ HEPES (Sigma-Aldrich), $120 \mathrm{mM} \mathrm{NaCl}$ (Merck, Darmstadt, Germany), $4.8 \mathrm{mM} \mathrm{KCl}$ (Sigma-Aldrich), $1 \mathrm{mM} \mathrm{CaCl} \bullet 2 \mathrm{H}_{2} \mathrm{O}$ (Sigma-Aldrich), $4.5 \mathrm{mM}$ glucose (Merck), $1.5 \%$ albumin (Sigma-Aldrich), and $0.2 \%$ collagenase II (Roche, Rotkreuz, Switzerland). The stromal vascular fraction was collected after filtration and centrifugation and subsequently cultivated as described for calvarial cells below.

Bone marrow stromal cells were isolated from femur and tibia of Men $1^{\text {gtRosaCreERT2 }}$ mice (6-8 weeks). Bone marrows were flushed out and cells were seeded at a density of $1 \times 10^{6}$ cells per $\mathrm{cm}^{2}$. After $72 \mathrm{~h}$, cells were washed three times with medium (DMEM containing $20 \%$ fetal bovine serum and $1 \%$ penicillin/streptomycin) and subsequently cultivated as described for calvarial cells below.

Primary osteoblasts isolated from calvaria of neonatal Men $1^{\text {gtRosaCreERT2 }}$ mice (postnatal days 3-5) by sequential digestions were cultivated as previously described. ${ }^{14}$ The cells were exposed to $1 \mu \mathrm{M} 4-\mathrm{OHT}$ (Sigma-Aldrich) for 3 days and subsequently subjected to osteogenic induction medium $(\alpha$-MEM containing $10 \%$ fetal bovine serum and $1 \%$ penicillin/streptomycin, supplemented with $100 \mathrm{mg} / \mathrm{ml}$ sodium ascorbate and $5 \mathrm{mM} \beta$-glycerophosphate; Sigma-Aldrich) and $/ 0 \mathrm{r} 100 \mathrm{ng} / \mathrm{ml}$ hBMP2 (R\&D Systems, Minneapolis, MN, USA). Osteoblast differentiation was determined by alkaline phosphatase and alizarin red (both from Sigma-Aldrich) staining at indicated time points.

Osteoclast differentiation. BMCs were isolated from femurs of 2- to 3-month-old mice and 200000 cells (diameter $>5 \mu \mathrm{m}$ ) were seeded per well in 96-well plates. Differentiation of osteoclasts was performed in $\alpha$-MEM containing $10 \%$ fetal bovine serum and $1 \%$ penicillin/streptomycin, supplemented with $50 \mathrm{ng} / \mathrm{ml}$ RANKL (PeproTech, Rocky Hill, NJ, USA) and $20 \mathrm{ng} / \mathrm{ml}$ M-CSF (R\&D Systems) for 5 or 7 days, with the medium changed every 2 or 3 days. TRAP staining was performed using a TRAP-kit (Sigma-Aldrich) and TRAP-positive cells with more than three nuclei were counted as osteoclasts. Osteoclast area was quantified using Osteomeasure software.

For conditioned medium-transfer experiments, conditioned medium from Men1deficient primary osteocyte-enriched fractions or control primary osteocyte-enriched fractions was diluted 1:1 in culture medium.

Osteocyte isolation. Primary osteocyte-enriched fractions from Men $1^{\text {gtRosaCreERT2 }}$ mice were isolated as previously described. ${ }^{27}$ Osteocytes were extracted by sequential digestions with collagenase and EDTA (AppliChem, Darmstadt, Germany). Fractions 3-5 of the sequential digest were considered as osteoblasts. Fractions 7-9 and cells derived from bone particles were pooled and considered as osteocytes. After one passage, 8000 cells per well were seeded into collagen-coated 96-well plates (Greiner Bio-One, Kremsmünster, Austria) and treated with $1 \mu \mathrm{M} \mathrm{4-OHT}$ for 3 days.

Co-culture experiments. BMCs were isolated from wild-type mice and 200000 cells per well were added on top of primary osteocyte-enriched fractions or primary osteoblasts cultivated in co-culture medium ( $\alpha$-MEM supplemented with $10 \mathrm{nM}$ 1,25-dihydroxyvitamin D3 (Sigma-Aldrich) and $20 \mathrm{ng} / \mathrm{ml} \mathrm{M-CSF).} \mathrm{For} \mathrm{pit}$ assay, bone slices (Immunodiagnostic Systems, Tyne and Wear, UK) were used. Cells were removed from bone slices with $1 \mathrm{M}$ ammonium hydroxide overnight and the resorption pits were visualized using $0.5 \%\left(\mathrm{w} / \mathrm{v}\right.$ in $\left.\mathrm{H}_{2} \mathrm{O}\right)$ toluidine blue.

For conditioned medium-transfer experiments, conditioned medium from Men1deficient primary osteocyte-enriched fractions or control primary osteocyte-enriched fractions was diluted 1:1 in co-culture medium. 
For neutralizing antibody experiments, CXCL10-neutralizing antibody $(20 \mathrm{ng} / \mathrm{ml}$; R\&D Systems) or IgG was added to co-cultures of wild-type BMCs with Men1deficient primary osteocyte-enriched fractions in co-culture medium.

The medium was changed every 3 days. After 9 days, cells were stained for TRAP activity.

Sorting of GFP+ primary osteocytes from Men1 ${ }^{\text {Dmp1Cre }} \times$ Rosa $^{\mathrm{mT} / \mathrm{mG}}$ mice. Primary osteocyte-enriched fractions were isolated from heterozygous (Men1 flox/+) or homozygous (Men1 floxflox) Men1 ${ }^{\text {Dmp1Cre, }}$ Rosa ${ }^{m T / m G}$ mice, and GFP+ cells were sorted using a FACS LSR II and FACSAria II (BD Biosciences, San Jose, CA, USA). GFP+ cells were used for RNA isolation (see below).

Calvarium ex vivo culture. Ex vivo organ culture from neonatal mouse calvaria was performed as previously described. ${ }^{28,29}$ Bone explants from calvaria were obtained by dissecting parietal bones and frontal bones from 7-day-old Men $1^{\text {flox }}$ or Men $1^{\text {Dmpicre }}$ mice. Calvaria were cut through the sagittal suture and each half was cultured in one well of a 24-well plate. Calvarial halves were treated with $1 \mu \mathrm{M}$ indomethacin (SigmaAldrich) in $\alpha$-MEM supplemented with fatty acid-free serum albumin (Sigma-Aldrich). After overnight incubation, calvarial halves were treated with CXCL10-neutralizing antibody (20 ng/ml) or lgG in $\alpha$-MEM supplemented with $10 \mathrm{nM} \mathrm{1,25-dihydroxyvitamin}$ D3 for 5 days. Medium was collected for CTX enzyme-linked immunosorbent assay (ELISA) and calvarial halves were used for RNA isolation or histology.

CXCL10-neutralizing antibody injection in vivo. Mouse CXCL10neutralizing antibody (R\&D Systems) or IgG (R\&D Systems) was delivered in the space between the subcutaneous tissue and the periosteum over the sagittal suture of the skull. Before the injection, all animals were anesthetized. One dose of CXCL10neutralizing antibody (100 $\mu \mathrm{g} /$ mouse) or lgG (100 $\mu \mathrm{g} /$ mouse), each in a $100 \mu \mathrm{l}$ volume, was injected subcutaneously. Mice were killed 5 days after the injection.

RNA isolation and QRT-PCR. Calvaria from 3-day-old mice were immersed in RLT buffer (Qiagen, Hilden, Germany) and subsequently homogenized using a Precellys 24 homogenizer (Peqlab, Erlangen, Germany). After centrifugation for 3 min at $10000 \times g$ at $4{ }^{\circ} \mathrm{C}$, the supernatant was used for total RNA isolation using RNeasy kit according to the manufacturer's instructions (Qiagen).

Primary osteoblasts or primary osteocyte-enriched fractions were lysed and total RNA was isolated (Qiagen). RNA was reverse-transcribed by a cDNA kit (Applied Biosystems, Carlsbad, CA, USA) and real-time qPCR was performed as previously described. ${ }^{30}$ Primer sequences are available upon request.

Immunoblotting. Primary osteoblasts stimulated with either hBMP2 $(100 \mathrm{ng} / \mathrm{ml})$ or hTGF $\beta 1$ ( $1 \mathrm{ng} / \mathrm{ml}$; PeproTech) following starvation in serum-free medium for $6 \mathrm{~h}$ were lysed in lysis buffer (Cell Signaling Technology, Danvers, MA, USA) containing proteinase inhibitor (Roche). Vertebra samples (L5) were pulverized using a mortar and pestle, and suspended in the lysis buffer. Total protein amount was determined by BCA assay (Pierce, Waltham, MA, USA) and immunoblotting was performed with antibodies against pSmad1/5/8 (1:1000; \#9511s, Cell Signaling Technology), Smad1/5/8 (1:1000; sc-6031, Santa Cruz Biotechnology, Dallas, TX, USA), pSmad3 (1:1000; \#9520, Cell Signaling Technology), Smad3 (1:1000; \#9523, Cell Signaling Technology), menin (1:10 000; A300-105A, Bethyl Laboratories, Montgomery, TX, USA), and $\beta$-actin (1:1000; sc-1616, Santa Cruz Biotechnology). Band intensity was analyzed using ImageJ software (NIH, Bethesda, MD, USA).

Enzyme-linked immunosorbent assay. Blood was collected into heparincoated tubes (Sarstedt, Nümbrecht, Germany) and kept at room temperature for at least $30 \mathrm{~min}$ before centrifugation at $2000 \times \mathrm{g}$ at room temperature for $10 \mathrm{~min}$ and isolation of the supernatant. PINP ELISA (Immunodiagnostic Systems) were performed with serum, and CTX ELISA (Immunodiagnostic Systems) was performed with serum and conditioned medium, according to the instructions of the manufacturers.

Immunohistochemistry. Paraffin sections ( $5 \mu \mathrm{m}$ thickness) of decalcified femurs were subjected to immunohistochemistry as previously described. ${ }^{13,31}$ Antigen retrieval was performed with antigen unmasking solution (Vector Laboratories, Peterborough, UK) at $95{ }^{\circ} \mathrm{C}$ for $10 \mathrm{~min}$ and immunohistochemistry was performed with an antibody against menin (1:3000; A300-105A, Bethyl Laboratories) and a biotinylated secondary antibody (1:200; Vector Laboratories).

Immunofluorescent staining. Cryosections (100 $\mu \mathrm{m}$ thickness) of decalcified femurs were subjected to immunohistochemistry as described elsewhere. ${ }^{32}$
Osteocalcin antibody (1:1000; LifeSpan BioSciences, Inc., Seattle, WA, USA) and Alexa Fluor 647 secondary antibody (1:200; Thermo Fisher Scientific, Waltham, MA, USA) were used. A Leica TCS-SP8 confocal microscope with an inverted stand (DMi8) and LAS X Software were used for imaging (Leica, Wetzlar, Germany). Images were taken at 40-fold magnification using a Leica HCX PL FL L 40×/0.60 objective.

Men1 overexpression in MLO-Y4 osteocyte cell line. RIKEN fulllength Mus musculus Men1 cDNA (F630025E01, Source BioScience, Nottingham, UK) was cloned into a modified lentiviral pLVX-IRES-GFP vector (Clontech Laboratories, Mountain View, CA, USA). The pLVX-IRES-Men1-GFP vector or pLVX-IRES-empty-GFP vector was co-transfected with psPax2 vector and pMD2.G vector into LentiX HEK 293T cells using CalPhos Mammalian Transfection Kit (Clontech Laboratories). Lentiviruses were collected and the MLO-Y4 osteocyte cell line was transduced according to the manufacturer's instructions. At 5 days after transduction, the GFP-expressing cells were sorted using a BD FACSARIA III (BD Biosciences) and expanded for further experiments.

Microarray analysis. Total RNAs isolated from primary osteocyte-enriched fractions $(80 \mathrm{ng})$ were processed and labeled for array hybridization using the Ambion WT Expression Kit (Life Technologies, Carlsbad, CA, USA) at the Genomics Core Facility, EMBL Heidelberg, Germany. Labeled, fragmented CDNA (Affymetrix GeneChip WT Terminal Labeling and Controls Kit, Santa Clara, CA, USA) was hybridized to Mouse Gene 2.0 arrays for $16 \mathrm{~h}$ at $45^{\circ} \mathrm{C}$ (Affymetrix GeneChip Hybridization, Wash, and Stain Kit). Arrays were washed and stained using the Affymetrix Fluidics Station 450, and scanned using the GeneArray Scanner 3000 7G (Affymetrix). Data analysis was performed with software Expression Console (Affymetrix). The estimated probe signals were normalized using the 'Robust Multiarray Average' method, and the expression levels of probe sets were generated in log2 scale that were then assigned to the annotated gene data (41 346 gene data sets). Probe sets with high expression levels ( $>50 \%$ of the total probe signals) were selected (19 854 gene data sets). These were submitted to DAVID Bioinformatics Resources (NIH) and annotated secreted factors were identified ( 775 gene data sets).

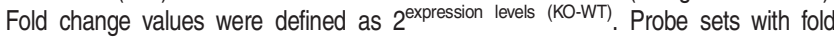
change values of $>1.4$ (30 gene data sets) or $<0.7$ ( 81 gene data sets) were selected as possible candidate genes for further validation.

Statistical analysis. Data are presented as mean \pm standard errors (S.E.M.). Statistical evaluations of two group comparisons were performed using Student's $t$-test. Statistical evaluations of experiments with more than two groups were performed using one-way analysis of variance (ANOVA).

\section{Conflict of Interest}

The authors declare no conflict of interest.

Acknowledgements. We are grateful to the staff of the animal facilities of the University of Ulm, led by Dr Petra Kirsch and in particular to Tobias Rappold. We thank Jelena Pistolic and Dr Vladimir Benes from the Genomics Core Facility, EMBL Heidelberg. We thank Dr. Lynda Bonewald for providing MLO-Y4 cells. We also thank Carmen Hamp and Christina Grimm for assistance in expression studies, and Susanne Schmidt for technical assistance. This study was supported by the Boehringer Ingelheim Foundation (to JPT), Deutsche Forschungsgemeinschaft (DFG, Priority Program Immunobone 1468, Tu 220/6-1, 6-2, Collaborative Research Center 1149, C02/INST 40/492-1, Trilateral Consortium Tu 220/12-1 to JPT), and Leibniz Graduate School on Aging, Fritz Lipmann Institute Jena (to SL).

\section{Author contributions}

$\mathrm{PL}, \mathrm{SL}, \mathrm{JK}, \mathrm{AR}, \mathrm{SO}, \mathrm{JL}, \mathrm{NM}, \mathrm{MMZ}, \mathrm{MN}, \mathrm{RW}$ and MR performed experiments, analyzed data, and contributed to the preparation of the manuscript. UHL, J-PD, PB, $\mathrm{CXZ}$, and JPT analyzed data and contributed to the preparation of the manuscript.

1. Xiong J, Onal M, Jilka RL, Weinstein RS, Manolagas SC, O'Brien CA. Matrix-embedded cells control osteoclast formation. Nat Med 2011; 17: 1235-U1262.

2. Nakashima T, Hayashi M, Fukunaga T, Kurata K, Oh-hora M, Feng JQ et al. Evidence for osteocyte regulation of bone homeostasis through RANKL expression. Nat Med 2011; 17: 1231-1234.

3. Dallas SL, Prideaux M, Bonewald LF. The osteocyte: an endocrine cell ... and more. Endocr Rev 2013; 34: 658-690. 
4. Ben-awadh AN, Delgado-Calle J, Tu X, Kuhlenschmidt K, Allen MR, Plotkin LI et al. Parathyroid hormone receptor signaling induces bone resorption in the adult skeleton by directly regulating the RANKL gene in osteocytes. Endocrinology 2014; 155: 2797-2809.

5. Wijenayaka AR, Kogawa M, Lim HP, Bonewald LF, Findlay DM, Atkins GJ. Sclerostin stimulates osteocyte support of osteoclast activity by a RANKL-dependent pathway. PLoS One 2011; 6: e25900.

6. Yang J, Shah R, Robling AG, Templeton E, Yang H, Tracey KJ et al. HMGB1 is a bone-active cytokine. J Cell Physiol 2008; 214: 730-739.

7. Kanazawa I, Canaff L, Abi Rafeh J, Angrula A, Li J, Riddle RC et al. Osteoblast menin regulates bone mass in vivo. J Biol Chem 2015; 290: 3910-3924.

8. Aziz A, Miyake T, Engleka KA, Epstein JA, McDermott JC. Menin expression modulates mesenchymal cell commitment to the myogenic and osteogenic lineages. Dev Biol 2009. 332: 116-130

9. Dreijerink KMA, Varier RA, van Beekum O, Jeninga EH, Hoppener JWM, Lips CJM et al. The multiple endocrine neoplasia type 1 (MEN1) tumor suppressor regulates peroxisome proliferator-activated receptor gamma-dependent adipocyte differentiation. Mol Cell Biol 2009; 29: 5060-5069.

10. Hendy GN, Kaji H, Sowa H, Lebrun JJ, Canaff L. Menin and TGF-beta superfamily member signaling via the smad pathway in pituitary, parathyroid and osteoblast. Horm Metab Res 2005; 37: 375-379

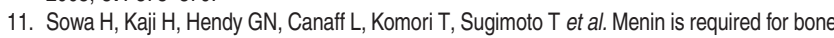
morphogenetic protein 2- and transforming growth factor beta-regulated osteoblastic differentiation through interaction with Smads and Runx2. J Biol Chem 2004; 279 40267-40275.

12. Sowa H, Kaji H, Canaff L, Hendy GN, Tsukamoto T, Yamaguchi T et al. Inactivation of menin, the product of the multiple endocrine neoplasia type 1 gene, inhibits the commitment of multipotential mesenchymal stem cells into the osteoblast lineage. J Biol Chem 2003; 278: 21058-21069.

13. Bertolino P, Tong WM, Herrera PL, Casse H, Zhang CX, Wang ZQ. Pancreatic beta-cellspecific ablation of the multiple endocrine neoplasia type 1 (MEN1) gene causes full penetrance of insulinoma development in mice. Cancer Res 2003; 63: 4836-4841.

14. Rauch A, Seitz S, Baschant U, Schilling AF, Illing A, Stride B et al. Glucocorticoids suppress bone formation by attenuating osteoblast differentiation via the monomeric glucocorticoid receptor. Cell Metab 2010; 11: 517-531.

15. Muzumdar MD, Tasic B, Miyamichi K, Li L, Luo L. A global double-fluorescent cre reporter mouse. Genesis 2007; 45: 593-605.

16. Clausen BE, Burkhardt C, Reith W, Renkawitz R, Forster I. Conditional gene targeting in macrophages and granulocytes using LysMcre mice. Transgen Res 1999; 8: 265-277.

17. Rodda SJ, McMahon AP. Distinct roles for Hedgehog and canonical Wnt signaling in specification, differentiation and maintenance of osteoblast progenitors. Development 2006; 133: 3231-3244

18. Lu Y, Xie Y, Zhang S, Dusevich V, Bonewald LF, Feng JQ. DMP1-targeted Cre expression in odontoblasts and osteocytes. J Dent Res 2007; 86: 320-325.

19. Huang DW, Sherman BT, Lempicki RA. Systematic and integrative analysis of large gene lists using DAVID bioinformatics resources. Nat Protoc 2009; 4: 44-57.

20. Lee J-H, Kim H-N, Kim K-O, Jin WJ, Lee S, Kim H-H et al. CXCL10 promotes osteolytic bone metastasis by enhancing cancer outgrowth and osteoclastogenesis. Cancer Res 2012; 72: 3175-3186.

21. Kittan NA, Allen RM, Dhaliwal A, Cavassani KA, Schaller M, Gallagher KA et al. Cytokine induced phenotypic and epigenetic signatures are key to establishing specific macrophage phenotypes. PLOS One 2013; 8: 15.
22. Yellin M, Paliienko I, Balanescu A, Ter-Vartanian S, Tseluyko V, Xu L-A et al. A phase II, randomized, double-blind, placebo-controlled study evaluating the efficacy and safety of MDX-1100, a fully human anti-CXCL10 monoclonal antibody, in combination with methotrexate in patients with rheumatoid arthritis. Arthritis Rheum 2012; 64: $1730-1739$.

23. Coutinho FL, Lourenco DM Jr, Toledo RA, Montenegro FLM, Correia-Deur JEM, Toledo SPA Bone mineral density analysis in patients with primary hyperparathyroidism associated with multiple endocrine neoplasia type 1 after total parathyroidectomy. Clin Endocrinol 2010; 72 : 462-468.

24. Dempster DW, Compston JE, Drezner MK, Glorieux FH, Kanis JA, Malluche $\mathrm{H}$ et al. Standardized nomenclature, symbols, and units for bone histomorphometry: a 2012 update of the report of the ASBMR Histomorphometry Nomenclature Committee. J Bone Miner Res 2013; 28: 1-16.

25. Parfitt AM, Drezner MK, Glorieux FH, Kanis JA, Malluche $H$, Meunier PJ et al. Bone histomorphometry: standardization of nomenclature, symbols, and units. J Bone Miner Res 1987; 2: 595-610.

26. Amling M, Priemel M, Holzmann T, Chapin K, Rueger JM, Baron R et al. Rescue of the skeletal phenotype of vitamin $\mathrm{D}$ receptor-ablated mice in the setting of normal mineral ion homeostasis: formal histomorphometric and biomechanical analyses. Endocrinology 1999; 140: $4982-4987$.

27. Stern AR, Stern MM, Van Dyke ME, Jaehn K, Prideaux M, Bonewald LF. Isolation and culture of primary osteocytes from the long bones of skeletally mature and aged mice. Biotechniques 2012; 52: 361-373.

28. Ljunggren $\mathrm{O}$, Ransjo $\mathrm{M}$, Lerner $\mathrm{UH}$. In vitro studies on bone resorption in neonatal mouse calvariae using a modified dissection technique giving four samples of bone from each calvaria. J Bone Miner Res 1991; 6: 543-550.

29. Lerner UH. Modifications of the mouse calvarial technique improve the responsiveness to stimulators of bone resorption. J Bone Miner Res 1987; 2: 375-383.

30. Tuckermann JP, Kleiman A, Moriggl R, Spanbroek R, Neumann A, Illing A et al. Macrophages and neutrophils are the targets for immune suppression by glucocorticoids in contact allergy. J Clin Invest 2007; 117: 1381-1390.

31. Bertolino P, Radovanovic I, Casse H, Aguzzi A,Wang ZQ, Zhang CX. Genetic ablation of the tumor suppresor menin causes lethality at mid-gestation with defects in multiple organs. Mech Develop 2003; 120: 549-560.

32. Ramasamy SK, Kusumbe AP, Wang L, Adams RH. Endothelial Notch activity promotes angiogenesis and osteogenesis in bone. Nature 2014; 507: 376-380.

(c) (1) (2) (2) This work is licensed under a Creative Commons Attribution-NonCommercial-ShareAlike 4.0 International License. The images or other third party material in this article are included in the article's Creative Commons license, unless indicated otherwise in the credit line; if the material is not included under the Creative Commons license, users will need to obtain permission from the license holder to reproduce the material. To view a copy of this license, visit http://creativecommons.org/licenses/by-nc-sa/4.0/

(C) The Author(s) 2017

Supplementary Information accompanies this paper on Cell Death and Differentiation website (http://www.nature.com/cdd) 